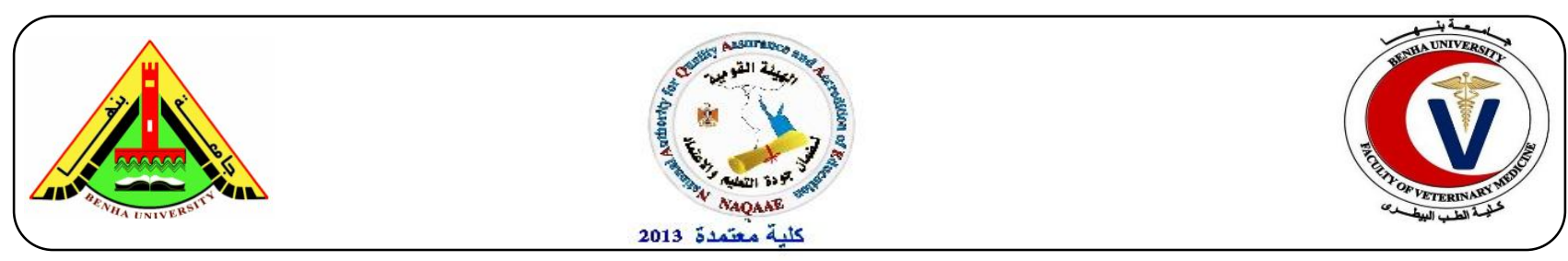

\title{
Effect of Some Plant Extract on Oxidative and Microbial Stability of Meat
}

\author{
Amani M. Salem ${ }^{1}$, Nahla A.Shawky ${ }^{2}$, Lamiaa M. Abo-Hussein ${ }^{2}$ \\ ${ }^{1}$ Food Control Dep., Fac. Vet. Med., Benha Univ. \\ ${ }^{2}$ Animal Health research institute, shebin el koom branch.
}

\section{A B S T R A C T}

The study was designed to investigate antimicrobial and antioxidant activity of olive leaves , curcuma and ginger extracts on minced meat samples. Minced meat was divided into 4 groups, untreated (control) and 3 treated groups, the first treated group was homogenized with olive leaves extract $(5 \% \& 3 \%) \&$ second group was homogenized with curcuma extract $(1 \%, 2 \%)$ and third group was homogenized with ginger extract $(0.4 \%, 0.6 \%)$. The minced meat samples were examined physically, bacteriologically and chemically to determine their keeping quality by aerobic plate, Staphylococcal and Enterobacteriacae counts, as well as measuring pH, TBA and TVN immediately after preparation (zero time) until 6 days of chilling storage $\left(4 \pm 1^{\circ} \mathrm{C}\right)$. Olive leaves extracts were more effective at all concentrations followed by ginger then curcuma extracts. Which may be due to flavonoids and phenolic compounds obtained from olive leaf extracts that play an important role as antioxidant and antimicrobial agents in the stored meat .

Key words: Olive leaves, ginger, curcuma, antimicrobial, antioxidant, minced meat.

(http://www.bvmj.bu.edu.eg)

(BVMJ-34(2): 8-22, 2018)

\section{INTRODUCTION}

Meat and meat products are susceptible to variation in quality parameters and deterioration of their nutritional components (Shah et al., 2014). Meat is an important source for some micronutrients such as trace elements and vitamins. In addition, meat as a protein-rich and carbohydrate-low product contributes to a low glycemic index, which is assumed to be beneficial with respect to obesity, diabetes development and cancer (Biesalski, 2005). However, meat get easily contaminated by microorganisms present in animal prior to slaughter. It is therefore important to make meat safe for consumers in terms of stability, transportation and storage.
Shelf-life and maintenance of meat quality are influenced by a number of interrelated factors including holding temperature, which can result in determinable changes in the quality attributes of meat. Spoilage by microbial growth is the most important factor in relation to keeping quality of meat (Lambert et al., 1991).

Moreover, spoilage of meat has remained a serious challenge in developing countries, including Egypt for decades, this has been due to poor storage systems in such countries where necessary facilities that could help to promote preservation are unavailable. (Olaoye and Onilude, 2010). 
Lipid oxidation and the growth of undesirable microorganisms in food products results in the development of spoilage, off flavor, rancidity, and deterioration, rendering such products unacceptable for human consumption (Mielnik et al., 2008). An increased interest has been directed towards plant-based extracts as source of phenolic antioxidants and antimicrobials (Skerget et al., 2005). Recently, the use of plant extracts as natural antioxidants has gained increasing interest because of the global trend of restriction in use of synthetic substances, also antioxidant rich plant extracts have potential benefits in food preservations (Uhart et al., 2006). Some studies expressed that plant extracts and EO components appear to make the cell membrane permeable and are able to disintegrate the outer membrane of Gramnegative bacteria .These are slightly more active against Gram-positive than Gramnegative bacteria.( Abdollahzadeh et al., 2014)

Olive leaf extract (OLE) has both antimicrobial and antioxidant activities due to its polyphenols (Lee and Lee, 2010).

Turmeric (Curcuma longa) is extensively used as spice, food preservative and coloring material in India, China and South East Asia. curcuminoids has been isolated from the rhizome of $\mathrm{C}$ longa, attributing a wide array of biological activities (Tilak et al. 2004; Kumar et al. 2006) Curcumin is a phenolic component obtained from turmeric (Sharma et al., 2005) curcumin has antioxidant, antimicrobial, anti-cancer and antiinflammatory effects (Aggarwal et al. 2007) .

Also, Ginger is one of the most used condiments for preparation of various dishes in India. (Fijelu Frank et al., 2014 ) suggested that ginger as a natural herb, could be used to extend the shelf life of meat products, providing the consumer with food containing natural additives, which might be seen more healthful than those of synthetic origin.
Therefore, this study was performed to investigate the antioxidant as well as the antimicrobial effectiveness of Olive leaves, ginger and curcuma extracts at various concentrations on the quality of fresh minced meat during chill storage $\left(4 \pm 1^{\circ} \mathrm{C}\right)$.

\section{MATERIALS AND METHODS}

\subsection{Collection of samples:}

About $5 \mathrm{~kg}$ fresh minced beef samples were collected from different butchers shops at El Menofia governorate and transported in an ice box as rapidly as possible to the laboratory. Minced meat samples were divided into 4 groups, untreated (control 200 gm) and 3 treated groups each one $400 \mathrm{gm}$ which were homogenized with olive leaves extract $(5 \% \mathrm{Vol} / \mathrm{W} \& 3 \% \mathrm{Vol} / \mathrm{W})$, turmeric extract $(1 \% \mathrm{Vol} / \mathrm{W} \& 2 \% \mathrm{Vol} / \mathrm{W})$ and ginger extract $(0.4 \% \mathrm{Vol} / \mathrm{W} \& 0.6 \% \mathrm{Vol} / \mathrm{W})$. Both untreated and treated samples were packaged in a separate plastic bags, stored at $\left(4 \pm 1^{\circ} \mathrm{C}\right)$ and then examined sensory, bacteriologically and chemically after ( $2 \mathrm{hrs}$ ) as zero time and at predetermined interval 24 hours. The experiment was conducted in triplicate and subjected to:

\subsection{Sensory analyses:}

The overall acceptability were determined for each sample of minced meat according to (Pearson and Tauber, 1984).

\subsection{Bacteriological analyses:}

Preparation of samples following ICMSF, 1978 was applied as $10 \mathrm{~g}$ portion of each sample was aseptically weighed into $90 \mathrm{ml}$ of $0.1 \%$ peptone water in a sterile plastic bag, and then blended in a Stomacher $400 \mathrm{Lab}$ Blender for 30 seconds. Ten-fold serial dilutions were used for bacteriological examination. 
Determination of :-

Aerobic Plate Count (ICMSF., 1996).

Enterobacteriaceae count according to (Gork, (1976) .

Staphylococci count according to(ICMSF., (1996).

\subsection{Chemical analyses:}

Determination of $\mathrm{pH}$ (Pearson and Tauber, 1984), Total Volatile Nitrogen (TVN) following Food and Agriculture Organization (FAO, 1980). and Thiobarbituric acid number (TBA) following (Vyncke, 1970) were applied. All experiments were conducted in triplicate.

\subsection{Statistical Analysis:}

Was adapted according to Gomez and Gomez (1984).

\section{RESULTS}

The results achieved in table (1) showed that sensory analyses of control (untreated groups) remain accepted until $2^{\text {nd }}$ day while treated groups with olive leaf extract $(5 \%$ and $3 \%$ ) remain accepted until $6^{\text {th }}$ day and $5^{\text {th }}$ day. Also, ginger extract (0.6 $\%$ and $0.4 \%$ ) remain accepted until $5^{\text {th }}$ day and $4^{\text {th }}$ day respectively. Moreover, curcuma extract $(0.2 \%$ and $0.1 \%)$ remain accepted until $4^{\text {th }}$ day and $3^{\text {th }}$ day, respectively.

Table $(2,3,4)$ indicated that the untreated samples showed the highest APC, Enterobacteriaceae counts and Staphylococcus count (log. cfu/g) comparing to the treated ones. The APC, Enterobacteriaceae counts and Staphylococcus count were gradually decrease during cold storage for all samples with different ratios depending on the type and concentration of the extracts. The treated samples with olive leaf extract (5\% and $3 \%$ ) showed the lowest counts followed by ginger extract $(0.6 \%$ and $0.4 \%)$ at all days of cold storage.

Also, the results obtained in table (5) showed that the reduction percentage of APC, Enterobacteriaceae and Staphylococci counts. The highest reduction percentage were in olive leaf extract (5\%) which recorded $55.8 \%, 54.7 \%$ and $58.6 \%$ at 6 th day of cold storage.

It is evident from the present investigation that the differences in $\mathrm{pH}$ mean values between different treated and untreated samples were significant $(P<0.05)$ during storage at $\left(4 \pm 1^{\circ} \mathrm{C}\right)$ as shown in table (6). The results showed an increase in $\mathrm{pH}$ mean values in untreated groups and decrease in treated ones. The highest rates were found in control samples $(7.06 \pm 0.022)$ while, treated samples with olive leaf extract ( $5 \%$ and $3 \%$ ) were the lowest $(3.51 \quad \pm .044)$ and $(3.55 \quad \pm .042)$ respectively followed by ginger extract $(0.6 \%$

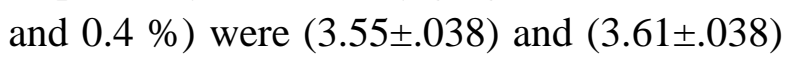
respectively, while , curcuma extract $(0.2 \%$ and $0.1 \%)$ were $(3.67 \pm .022)$ and $(3.71 \pm .021)$ at $6^{\text {th }}$ day of cold storage at $\left(4 \pm 1^{\circ} \mathrm{C}\right)$, respectively.

Table (7) showed an increase in TVN mean values $(\mathrm{mg} / 100 \mathrm{~g})$ in untreated groups and decrease in treated ones. The highest rates were found in control samples $(15.17 \pm .088)$ while, treated samples with olive leaf extract

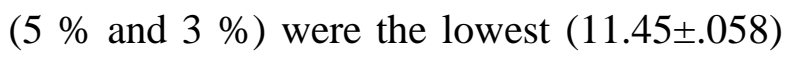
and $(11.48 \pm .088)$ respectively followed by ginger extract $(0.6 \%$ and $0.4 \%)$ were $(11.46 \pm .058)$ and $(11.51 \pm .058)$ respectively, while , curcuma extract $(0.2 \%$ and $0.1 \%)$ were $(11.59 \pm .033)$ and $(11.61 \pm .033)$ at $6^{\text {th }}$ day of cold storage at $\left(4 \pm 1^{\circ} \mathrm{C}\right)$, respectively.

On the other hand, TBA mean values in different treatments during storage by different rates shown in table (8) The untreated (control) sample showed the highest value $(0.599 \pm 0.006)$ in $6^{\text {th }}$ day of storage, while, treated samples with olive leaf extract 
(5 \% and $3 \%$ ) were the lowest $(0.223$ $\pm 0.009) \quad$ and $\quad(0.227 \pm 0.012)$

respectively followed by ginger extract $(0.6$ $\%$ and $0.4 \%)$ were $(0.226 \quad \pm 0.009) \quad$ and

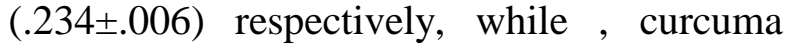
extract $(0.2 \%$ and $0.1 \%)$ were $(0.250 \pm 0.015)$ and $(0.260 \pm 0.006)$ at $6^{\text {th }}$ day of cold storage at $\left(4 \pm 1^{\circ} \mathrm{C}\right)$, respectively.

Table (1): Effect of various concentrations of natural extracts on overall acceptability of minced beef during cold storage at $4 \pm 1^{\circ} \mathrm{C}$.

Groups $\quad$ conc. $\quad \begin{gathered}\text { Zero } \\ \text { day }\end{gathered} 1^{\text {st }}$ day $\quad 2^{\text {nd }}$ day $\quad 3^{\text {rd }}$ day $\quad 4^{\text {th }}$ day $\quad 5^{\text {th }}$ day 6 th day

\begin{tabular}{|c|c|c|c|c|c|c|c|c|}
\hline Control & - & 9 & 7 & 5 & 3 & 2 & 1 & 1 \\
\hline \multirow[t]{2}{*}{ Olive leaf } & $5 \%$ & 9 & 9 & 8 & 7 & 6 & 6 & 5 \\
\hline & $3 \%$ & 9 & 9 & 7 & 6 & 6 & 5 & 4 \\
\hline \multirow[t]{2}{*}{ curcuma } & $2 \%$ & 9 & 8 & 7 & 6 & 5 & 3 & 2 \\
\hline & $1 \%$ & 9 & 7 & 6 & 5 & 4 & 2 & 2 \\
\hline \multirow[t]{2}{*}{ ginger } & $0.6 \%$ & 9 & 9 & 7 & 7 & 6 & 5 & 4 \\
\hline & $0.4 \%$ & 9 & 8 & 7 & 6 & 5 & 4 & 3 \\
\hline
\end{tabular}

Score System for Sensory Evaluation:

9: Excellent

8: Very very good

7: Very good

6: Good

5: Medium
4: Fair

3: Poor

2: Very poor

1 : Very very poor 
Table (2): The effects of various concentrations of natural extracts on APC (log. $\mathrm{cfu} / \mathrm{g}$ ) of the examined minced meat samples during cold storage at $4 \pm 1^{\circ} \mathrm{C}$.

\begin{tabular}{|c|c|c|c|c|c|c|c|}
\hline \multirow[t]{2}{*}{ Groups } & \multirow[t]{2}{*}{ control } & \multicolumn{2}{|c|}{ Olive leaf } & \multicolumn{2}{|c|}{ curcuma } & \multicolumn{2}{|l|}{ Ginger } \\
\hline & & $5 \%$ & $3 \%$ & $2 \%$ & $1 \%$ & $0.6 \%$ & $0.4 \%$ \\
\hline Zero & 5.72 & 5.72 & $5.72^{h}$ & 5.72 & $5.72 \mathrm{~h}$ & $5.72 \mathrm{~h}$ & 5.72 \\
\hline \multirow[t]{2}{*}{ day } & \pm & \pm & \pm & \pm & \pm & \pm & \pm \\
\hline & 4.75 & 4.75 & 4.75 & 4.75 & 4.75 & 4.75 & 4.75 \\
\hline \multirow[t]{3}{*}{$1^{\text {th }}$ day } & $5.84^{h}$ & $5.55^{h}$ & 5.67 & 5.68 & 5.69 & 5.67 & $5.68^{h}$ \\
\hline & \pm & \pm & \pm & \pm & \pm & \pm & \pm \\
\hline & 4.96 & 4.73 & 4.67 & 4.72 & 4.7 & 4.72 & 4.69 \\
\hline \multirow[t]{3}{*}{$2^{\text {rd }}$ day } & 6.86 & 5.41 & $5.66^{h}$ & 5.67 & $\mathrm{gh}_{5.68}$ & 5.66 & 5.67 \\
\hline & \pm & \pm & \pm & \pm & \pm & \pm & \pm \\
\hline & 5.53 & 4.44 & 5.34 & 5.08 & 4.76 & 5.4 & 5.47 \\
\hline \multirow[t]{3}{*}{$3^{\text {nd }}$ day } & 7.34 & 5.01 & 5.43 & 5.44 & $5.45^{\mathrm{fgh}}$ & $5.43^{\mathrm{fgh}}$ & 5.44 \\
\hline & \pm & \pm & \pm & \pm & \pm & \pm & \pm \\
\hline & 6.5 & 6.49 & 6.51 & 6.32 & 6.27 & 6.48 & 6.45 \\
\hline \multirow[t]{3}{*}{$4^{\text {st }}$ day } & $7.92^{f}$ & $4.71^{\mathrm{fgh}}$ & $4.74^{\mathrm{fgh}}$ & 4.78 & 4.79 & 4.74 & 4.76 \\
\hline & \pm & \pm & \pm & \pm & \pm & \pm & \pm \\
\hline & 6.63 & 6.49 & 6.52 & 6.44 & 6.44 & 6.4 & 6.44 \\
\hline \multirow[t]{3}{*}{$5^{\text {st }}$ day } & 8.14 & 4.35 & $4.72^{\mathrm{e}}$ & $4.76^{\mathrm{cd}}$ & 4.77 & 4.72 & 4.74 \\
\hline & \pm & \pm & \pm & \pm & \pm & \pm & \pm \\
\hline & 7.53 & 7.7 & 7.7 & 7.4 & 7.65 & 7.64 & 7.7 \\
\hline \multirow[t]{3}{*}{$6^{\text {st }}$ day } & 8.52 & 3.76 & 3.79 & 3.85 & $3.88^{b}$ & $3.77^{e}$ & 3.78 \\
\hline & \pm & \pm & \pm & \pm & \pm & \pm & \pm \\
\hline & 3.55 & 3.67 & 3.72 & 3.49 & 3.58 & 3.58 & 3.45 \\
\hline
\end{tabular}

The values represent mean \pm SD of three experiments.

Different letters within the same (column/row) indicate significant difference at $\mathrm{p}<0.05$ 
Table (3): The effects of various concentrations of natural extracts on Enterobacteriace $(\mathrm{log} . \mathrm{cfu} / \mathrm{g})$ of the examined minced meat samples during cold storage at $4 \pm 1^{\circ} \mathrm{C}$.

\begin{tabular}{crrrrrrr}
\hline Groups & control & \multicolumn{2}{c}{ Olive leaf } & \multicolumn{2}{c}{ curcuma } & & Ginger \\
\cline { 2 - 8 } zero & & $5 \%$ & $3 \%$ & $2 \%$ & $1 \%$ & $0.6 \%$ & $0.4 \%$ \\
\cline { 2 - 8 } day & 4.92 & 4.92 & $4.92^{\mathrm{e}}$ & 4.92 & 4.92 & $4.92^{\mathrm{e}}$ & 4.92 \\
& \pm & \pm & \pm & \pm & \pm & \pm & \pm \\
$1^{\text {th }}$ day & 4.14 & 4.14 & 4.14 & 4.14 & 4.14 & 4.14 & 4.14 \\
& $4.98^{\mathrm{e}}$ & $4.55^{\mathrm{e}}$ & 4.58 & 4.63 & 4.64 & 4.56 & $4.58^{\mathrm{e}}$ \\
& \pm & \pm & \pm & \pm & \pm & \pm & \pm \\
$2^{\text {rd }}$ day & 4.64 & 4.22 & 4.34 & 4.42 & 4.54 & 4.23 & 4.33 \\
& 5.12 & $4.24^{\mathrm{e}}$ & 4.31 & 4.34 & $4.48^{\mathrm{e}}$ & 4.31 & 4.35 \\
& \pm & \pm & \pm & \pm & \pm & \pm & \pm \\
$3^{\text {nd }}$ day & 4.67 & 3.95 & 3.81 & 3.75 & 3.47 & 3.07 & 3.12 \\
& 5.24 & 4.05 & $4.1^{\mathrm{e}}$ & 4.19 & $4.25^{\mathrm{e}}$ & $4.06^{\mathrm{e}}$ & 4.17 \\
$4^{\text {st }}$ day & \pm & \pm & \pm & \pm & \pm & \pm & \pm \\
& 4.61 & 3.6 & 3.68 & 3.75 & 3.77 & 3.61 & 3.62 \\
& $5.54^{\mathrm{e}}$ & $3.59^{\mathrm{e}}$ & 3.63 & 3.78 & 3.78 & 3.45 & 3.59 \\
$5^{\text {st }}$ day & \pm & \pm & \pm & \pm & \pm & \pm & \pm \\
& 5.38 & 3.14 & 3.13 & 3.28 & 3.42 & 3.13 & 3.17 \\
& 5.81 & 3.21 & $3.25^{\mathrm{cd}}$ & 3.34 & 3.42 & 3.24 & 3.21 \\
$6^{\text {st }}$ day & \pm & \pm & \pm & \pm & \pm & \pm & \pm \\
& 5.86 & 3.12 & 3.15 & 3.12 & 3.22 & 3.19 & 3.15 \\
& 5.99 & 2.71 & 2.78 & 2.81 & $2.87^{\mathrm{c}}$ & $2.74^{\text {cd }}$ & $2.71^{\text {cd }}$ \\
& \pm & \pm & \pm & \pm & \pm & \pm & \pm \\
& 5.89 & 2.25 & 2.29 & 2.35 & 2.35 & 2.25 & 2.3 \\
\hline
\end{tabular}

The values represent mean \pm SD of three experiments.

Different letters within the same (column/row) indicate significant difference at $\mathrm{p}<0.05$ 
Table (4): The effects of various concentrations of natural extracts on Staphylococci $(\mathrm{log}$. cfu $/ \mathrm{g}$ ) of the examined minced meat samples during cold storage at $4 \pm 1^{\circ} \mathrm{C}$.

\begin{tabular}{|c|c|c|c|c|c|c|c|}
\hline \multirow[t]{2}{*}{ Groups } & \multirow[t]{2}{*}{ control } & \multicolumn{2}{|c|}{ Olive leaf } & \multicolumn{2}{|c|}{ curcuma } & \multicolumn{2}{|c|}{ Ginger } \\
\hline & & $5 \%$ & $3 \%$ & $2 \%$ & $1 \%$ & $0.6 \%$ & $0.4 \%$ \\
\hline Zero & 4.53 & 4.53 & $4.53^{g}$ & 4.53 & 4.53 & $4.53^{g}$ & 4.53 \\
\hline \multirow[t]{2}{*}{ day } & \pm & \pm & \pm & \pm & \pm & \pm & \pm \\
\hline & 2.25 & 2.25 & 2.25 & 2.25 & 2.25 & 2.25 & 2.25 \\
\hline \multirow[t]{3}{*}{$1^{\text {th }}$ day } & $4.89^{g}$ & $4.33^{g}$ & 4.35 & 4.39 & 4.42 & 4.34 & $4.37^{\mathrm{g}}$ \\
\hline & \pm & \pm & \pm & \pm & \pm & \pm & \pm \\
\hline & 2.55 & 2.44 & 2.44 & 2.36 & 2.36 & 2.5 & 2.5 \\
\hline \multirow[t]{3}{*}{$2^{\text {rd day }}$} & 5.14 & 4.03 & $4.09^{g}$ & $4.11^{\mathrm{g}}$ & 4.21 & 4.09 & 4.12 \\
\hline & \pm & \pm & \pm & \pm & \pm & \pm & \pm \\
\hline & 3.32 & 3.4 & 3.38 & 3.18 & 3.18 & 3.42 & 3.45 \\
\hline \multirow[t]{3}{*}{$3^{\text {nd }}$ day } & 5.73 & 3.63 & 3.71 & $3.87^{g}$ & 3.88 & $3.71^{g}$ & 3.74 \\
\hline & \pm & \pm & \pm & \pm & \pm & \pm & \pm \\
\hline & 3.46 & 3.74 & 3.72 & 3.32 & 3.25 & 3.69 & 3.7 \\
\hline \multirow[t]{3}{*}{$4^{\text {st }}$ day } & 5.93 & $3.25^{f}$ & 3.31 & 3.45 & 3.49 & 3.26 & $3.32^{f}$ \\
\hline & \pm & \pm & \pm & \pm & \pm & \pm & \pm \\
\hline & 4.16 & 4.57 & 4.49 & 3.95 & 4.16 & 4.61 & 4.53 \\
\hline \multirow[t]{3}{*}{$5^{\text {st }}$ day } & 6.12 & $3.02^{f}$ & $3.11^{\text {ef }}$ & 3.23 & $3.25^{\mathrm{ef}}$ & 3.12 & 3.19 \\
\hline & \pm & \pm & \pm & \pm & \pm & \pm & \pm \\
\hline & 4.06 & 4.64 & 4.57 & 4.08 & 3.76 & 4.57 & 4.59 \\
\hline \multirow[t]{3}{*}{$6^{\text {st }}$ day } & 6.48 & 2.68 & 2.71 & $2.86^{b}$ & 2.91 & $2.67^{d}$ & 2.73 \\
\hline & \pm & \pm & \pm & \pm & \pm & \pm & \pm \\
\hline & 5.69 & 5.84 & 5.85 & 5.4 & 5.45 & 5.88 & 5.89 \\
\hline
\end{tabular}

The values represent mean \pm SD of three experiments.

Different letters within the same (column/row) indicate significant difference at $\mathrm{p}<0.05$ 
Table(5) Reduction percentage of microbial growth with different concentrations of natural extracts.

\begin{tabular}{|c|c|c|c|c|c|c|c|}
\hline M.O & Groups & $1^{\text {st }}$ & $2^{\text {nd }}$ & $3^{\text {rd }}$ & $4^{\text {th }}$ & $5^{\text {th }}$ & $6^{\text {th }}$ \\
\hline \multirow[t]{7}{*}{ APC } & Olive leaf $5 \%$ & $4.9 \%$ & $21.3 \%$ & 31.7 & $40.5 \%$ & $46.5 \%$ & $55.8 \%$ \\
\hline & & & & $\%$ & & & \\
\hline & Olive leaf $3 \%$ & $3.6 \%$ & $17.4 \%$ & $\begin{array}{c}26.0 \\
\%\end{array}$ & $40.1 \%$ & $42.0 \%$ & $55.5 \%$ \\
\hline & Curcuma $2 \%$ & $2.7 \%$ & $17.3 \%$ & $25.8 \%$ & $39.6 \%$ & $41.5 \%$ & $54.8 \%$ \\
\hline & Curcuma $1 \%$ & $2.5 \%$ & $17.2 \%$ & $\begin{array}{c}25.7 \\
\%\end{array}$ & $39.5 \%$ & $41.4 \%$ & $54.4 \%$ \\
\hline & Ginger $0.6 \%$ & $3.6 \%$ & $17.4 \%$ & $\begin{array}{c}26.0 \\
\%\end{array}$ & $40.1 \%$ & $42.0 \%$ & $55.7 \%$ \\
\hline & Ginger $0.4 \%$ & $2.7 \%$ & $17.3 \%$ & $25.8 \%$ & $39.8 \%$ & $41.7 \%$ & $55.6 \%$ \\
\hline \multirow[t]{6}{*}{ Enterobacteriacea } & Olive leaf $5 \%$ & $8.6 \%$ & $\begin{array}{c}17.1 .3 \\
\%\end{array}$ & $22.7 \%$ & $35.1 \%$ & $44.7 \%$ & $54.7 \%$ \\
\hline & Olive leaf $3 \%$ & $8.0 \%$ & $15.8 \%$ & $21.7 \%$ & $34.4 \%$ & $44.0 \%$ & $53.5 \%$ \\
\hline & Curcuma $2 \%$ & $7.0 \%$ & $15.2 \%$ & $20.0 \%$ & $31.7 \%$ & $42.5 \%$ & $53.0 \%$ \\
\hline & Curcuma $1 \%$ & $6.8 \%$ & $12.5 \%$ & $18.8 \%$ & $31.7 \%$ & $41.1 \%$ & $52.0 \%$ \\
\hline & Ginger $0.6 \%$ & $8.4 \%$ & $15.8 \%$ & $22.5 \%$ & $37.7 \%$ & $44.2 \%$ & $54.2 \%$ \\
\hline & Ginger $0.4 \%$ & $8.0 \%$ & $15.0 \%$ & $20.4 \%$ & $35.1 \%$ & $44.7 \%$ & $54.7 \%$ \\
\hline \multirow[t]{6}{*}{ Staphylococci } & Olive leaf $5 \%$ & $11.4 \%$ & $21.5 \%$ & $36.6 \%$ & $45.1 \%$ & $50.6 \%$ & $58.6 \%$ \\
\hline & Olive leaf $3 \%$ & $11.0 \%$ & $20.4 \%$ & $35.2 \%$ & $44.1 \%$ & $49.1 \%$ & $58.1 \%$ \\
\hline & Curcuma $2 \%$ & $10.2 \%$ & $20.0 \%$ & $32.4 \%$ & $41.8 \%$ & $47.2 \%$ & $55.8 \%$ \\
\hline & Curcuma $1 \%$ & $9.6 \%$ & $18.0 \%$ & $32.2 \%$ & $41.1 \%$ & $46.8 \%$ & $55.0 \%$ \\
\hline & Ginger $0.6 \%$ & $11.2 \%$ & $20.4 \%$ & $35.2 \%$ & $45.0 \%$ & $49.0 \%$ & $58.7 \%$ \\
\hline & Ginger $0.4 \%$ & $10.6 \%$ & $19.8 \%$ & $34.7 \%$ & $44.0 \%$ & $47.8 \%$ & $\mathbf{5 7 . 8 \%}$ \\
\hline
\end{tabular}


Table (6): The effects of various concentrations of natural extracts on $\mathrm{pH}$ of the examined minced meat samples stored at $4 \pm 1{ }^{\circ} \mathrm{C}$.

\begin{tabular}{|c|c|c|c|c|c|c|c|}
\hline Days & Control & $\begin{array}{c}\text { Olive leaf } \\
(5 \%)\end{array}$ & $\begin{array}{c}\text { Olive leaf } \\
(3 \%)\end{array}$ & $\begin{array}{c}\text { curcuma } \\
(2 \%)\end{array}$ & $\begin{array}{c}\text { curcuma } \\
(1 \%)\end{array}$ & $\begin{array}{l}\text { Ginger } \\
(0.6 \%)\end{array}$ & $\begin{array}{l}\text { Ginger } \\
(0.4 \%)\end{array}$ \\
\hline Zero & $5.66^{\mathrm{n}} \pm .117$ & $5.66 \pm .117$ & $5.66^{\mathrm{n}} \pm .117$ & $5.66 \pm .117$ & $5.66^{\mathrm{n}} \pm .117$ & $5.66 \pm .117$ & $5.66 \pm .117$ \\
\hline $1^{s t}$ & $5.89^{\mathrm{m}} \pm .038$ & $5.61 \pm .092$ & $5.66 \pm .086$ & $5.81 \pm .041$ & $5.87 \pm .033$ & $5.63^{n} \pm .085$ & $5.64 \pm .084$ \\
\hline $2^{\text {nd }}$ & $6.42 \pm .118$ & $5.21 \pm .064$ & $5.23^{1} \pm .064$ & $5.37 \pm .090$ & $5.38^{\mathrm{jk}} \pm .090$ & $5.23 \pm .061$ & $5.26 \pm .070$ \\
\hline $3^{\text {th }}$ & $6.77 \pm .030$ & $5.01 \pm .026$ & $5.09^{\mathrm{ij}} \pm .009$ & $5.19^{\mathrm{h}} \pm .021$ & $5.21 \pm .021$ & $5.03 \pm .030$ & $5.11 \pm .024$ \\
\hline $4^{\text {th }}$ & $6.95^{b c} \pm .023$ & $4.61 \pm .012$ & $4.64 \pm .021$ & $4.83 \pm .023$ & $4.86 \pm .021$ & $4.63^{\mathrm{def}} \pm .015$ & $4.65^{\mathrm{h}} \pm .015$ \\
\hline $5^{\text {th }}$ & $7.01 \pm .003$ & $4.15^{\mathrm{g}} \pm .023$ & $4.22^{\mathrm{fg}} \pm .015$ & $4.35 \pm .035$ & $4.37 \pm .017$ & $4.16 \pm .023$ & $4.24 \pm .024$ \\
\hline $6^{\text {th }}$ & $7.06 \pm .022$ & $3.51^{\mathrm{efg}} \pm .044$ & $3.55 \pm .042$ & $3.67 \pm .022$ & $3.71^{\mathrm{ab}} \pm .021$ & $3.55 \pm .038$ & $3.61 \pm .038$ \\
\hline
\end{tabular}

The values represent mean \pm SD of three experiments.

Different letters within the same (column/row) indicate significant difference at $\mathrm{p}<0.05$ 
Table (7): The effects of various concentrations of natural extracts on TVN (mg/100g) of the examined minced meat samples during cold storage at $4 \pm 1^{\circ} \mathrm{C}$.

\begin{tabular}{|c|c|c|c|c|c|c|c|}
\hline \multirow[t]{2}{*}{ Groups } & \multirow[t]{2}{*}{ control } & \multicolumn{2}{|r|}{ Olive leaf } & \multicolumn{2}{|r|}{ curcuma } & \multicolumn{2}{|r|}{ Ginger } \\
\hline & & $5 \%$ & $3 \%$ & $2 \%$ & $1 \%$ & $0.6 \%$ & $0.4 \%$ \\
\hline \multirow{3}{*}{$\begin{array}{l}\text { Zero } \\
\text { day }\end{array}$} & 13.37 & $13.37^{\text {st }}$ & 13.37 & 13.37 & $13.37^{\text {st }}$ & 13.37 & 13.37 \\
\hline & \pm & \pm & \pm & \pm & \pm & \pm & \pm \\
\hline & 0.24 & 0.24 & 0.24 & 0.24 & 0.24 & 0.24 & 0.24 \\
\hline \multirow[t]{3}{*}{$1^{\text {th }}$ day } & 13.53 & $13.01^{\mathrm{rst}}$ & 13.05 & 13.15 & $13.18^{\text {opqrs }}$ & 13.04 & $13.11^{\text {pqrs }}$ \\
\hline & \pm & \pm & \pm & \pm & \pm & \pm & \pm \\
\hline & 0.176 & 0.208 & 0.203 & 0.176 & 0.176 & 0.208 & 0.208 \\
\hline \multirow[t]{3}{*}{$2^{\text {rd day }}$} & $13.87^{\mathrm{jklmn}}$ & 12.87 & $12.89^{\text {Imnop }}$ & 12.93 & $12.97^{\mathrm{ijkl}}$ & 12.9 & 12.94 \\
\hline & \pm & \pm & \pm & \pm & \pm & \pm & \pm \\
\hline & 0.088 & 0.12 & 0.12 & 0.033 & 0.088 & 0.115 & 0.153 \\
\hline \multirow[t]{3}{*}{$3^{\text {nd }}$ day } & 14.1 & 12.43 & 12.51 & 12.6 & $12.65^{\text {defghi }}$ & 12.44 & 12.49 \\
\hline & \pm & \pm & \pm & \pm & \pm & \pm & \pm \\
\hline & 0.058 & 0.233 & 0.233 & 0.173 & 0.145 & 0.26 & 0.26 \\
\hline \multirow[t]{3}{*}{$4^{\text {st }}$ day } & $14.23^{\text {cdefgh }}$ & 12.02 & $12.09^{\text {hijk }}$ & 12.21 & 12.31 & $12.08^{\text {ghij }}$ & 12.17 \\
\hline & \pm & \pm & \pm & \pm & \pm & \pm & \pm \\
\hline & 0.033 & 0.12 & 0.058 & 0.058 & 0.067 & 0.133 & 0.12 \\
\hline \multirow[t]{3}{*}{$5^{\text {st }}$ day } & 14.87 & 11.87 & $11.89^{\text {defghi }}$ & 11.97 & 11.99 & 11.88 & 11.91 \\
\hline & \pm & \pm & \pm & \pm & \pm & \pm & \pm \\
\hline & 0.033 & 0.12 & 0.12 & 0.033 & 0.058 & 0.115 & 0.115 \\
\hline \multirow[t]{3}{*}{$6^{\text {st }}$ day } & 15.17 & $11.45^{\text {efghij }}$ & 11.48 & $11.59^{\text {cde }}$ & $11.61^{\mathrm{e}}$ & 11.46 & 11.51 \\
\hline & \pm & \pm & \pm & \pm & \pm & \pm & \pm \\
\hline & 0.088 & 0.058 & 0.088 & 0.033 & 0.033 & 0.058 & 0.058 \\
\hline
\end{tabular}

The values represent mean \pm SD of three experiments.

Different letters within the same (column/row) indicate significant difference at $\mathrm{p}<0.05$ 
Table (8): The effects of various concentrations of natural extracts on TBA (Melanoaldehyde / $\mathrm{Kg}$ ) of the examined minced meat samples during cold storage at $4 \pm 1^{\circ} \mathrm{C}$.

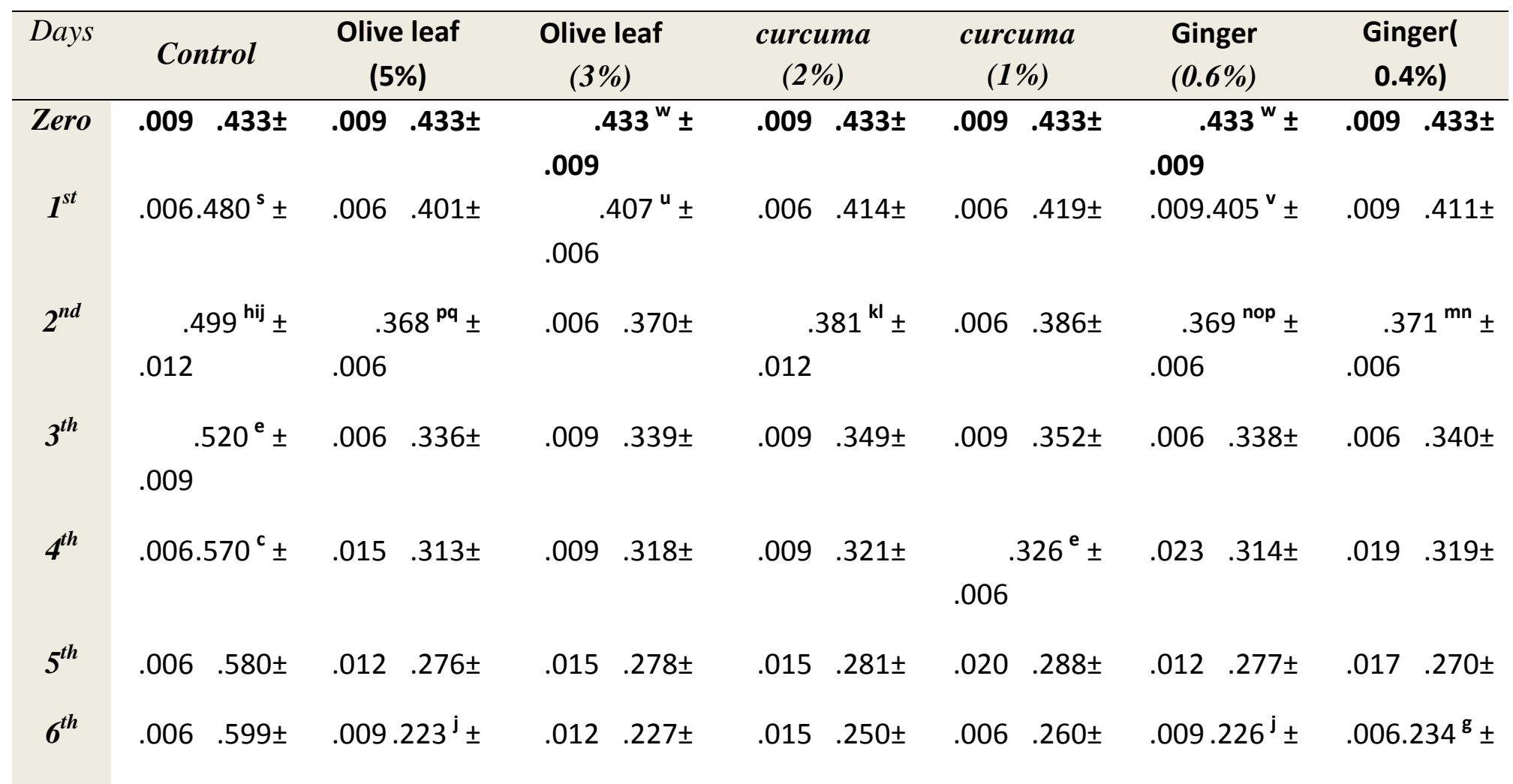

The values represent mean \pm SD of three experiments.

Different letters within the same (column/row) indicate significant difference at $\mathrm{p}<0.05$

\section{DISCUSSION}

Sensory evaluation is an easy, quick and efficient method for getting idea about the quality of the product and its overall acceptance; sensory methods were used to assess the degree of freshness based on organoleptic characteristics such as color, odor, texture and overall acceptability of the product (Haq et al., 2013). It is obvious from results obtained in table (1) that the sensory properties of different treated minced meat samples during cold storage $\left(4 \pm 1^{\circ} \mathrm{C}\right)$ were enhanced and shelf-life were extended .The obtained results indicated that the best sensory quality was attained at the highest concentration of olive leaf and ginger, while slight improvement in sensory quality of curcuma-treated minced beef samples was noticed in samples as compared with untreated samples. These results were nearly similar to those results obtained by Mancini et al., ( 2017 ) , Omojola et al., (2015) showed that the ethanolic extract of ginger had salutary effects on the sensory profile. Therefore it is suggested that ginger, extract as a natural herb could be used to extended the shelf-life of chicken patties provide the consumer with food containing natural additives which might be more healthful . 
Marangoni et al., (2017) cleared that the olive leaves delayed the formation of rancid flavors and odors and provided higher juiciness and lower acidity for chicken meat.

The results illustrated in table (2) agreed with those recorded by Soni et al.( 2006), where they found that, the olive extracted from the leaf have a great antimicrobial activities on bacteria found in meat. These effects have been attributed, in part, to the presence in the Mediterranean diet of antioxidant vitamins, flavanoids and polyphenols that play an important role in disease prevention (Briante et al., 2002; Benavente-Garcia et al., 2000). In other words, flavonoids and phenolic compounds obtained from olive leaf are known to have diverse biological activities and may also be responsible for the pharmacological actions of olive leaf or, at least synergistically reinforcing those actions (Burt et al., 2004). Also, $\mathrm{Du}$ and $\mathrm{Li}$, (2008) recorded that turmeric and ginger have antimicrobial activities as they reduce the number of total bacterial counts than the control group.

Also, Enterobacteriaceae count (log. $\mathrm{cfu} / \mathrm{g})$ decreased and this result agreed with those of Pattaratanawadee et al.( 2006) who found that olive leaf extract, turmeric and ginger are highly effective against $\mathrm{G}+\mathrm{ve}$ and $\mathrm{G}$-ve bacteria.

For APC, all treated samples were accepted according to (EOS, 2005) as permissible limit $\left(10^{6} / \mathrm{g}\right)$. While, Enterobacteriaceae and Staphylococci in both treated and untreated groups were unaccepted according to (EOS, 2005) permissible limit $\left(10^{2} / \mathrm{g}\right)$ for both.

As shown in table (4), the control samples had the highest counts of Staphylococcus count and this nearly similar to those obtained by (Calo et al., 2015) who found that, The $G+v e$ bacteria are sensitive to polyphenols since the bacterial membranes interact with hydrophobic components of the polyphenols. On the other hand, G -ve bacteria are more resistant to polyphenols because they possess a hydrophilic cell wall. The reduction of $\mathrm{G}+\mathrm{ve}$ bacteria with addition of olive oils than that of $\mathrm{G}-\mathrm{ve}$ bacteria due to the gram +ve highly sensitive to polyphenyls found in olive and ginger.

$\mathrm{pH}$ plays an important role for microbiological growth affecting shelf-life of the meat products (Amal and Soher, 2010). Table (6) showed $\mathrm{pH}$ mean values and agree with those of Aytul (2010) who found that, $\mathrm{pH}$ values of all samples decreased up to the 6 th day of storage. However, at the 9th day $\mathrm{pH}$ values increased except for the sample treated with $2 \%$ OLE. Control samples had the lowest $\mathrm{pH}$ values compared to the samples treated with OLE up to the 6th day, but at the 9th day control samples had the highest $\mathrm{pH}$ values. No particular trend was observed among the $\mathrm{pH}$ values of control and treated samples.

Zemenu (2017) found that $\mathrm{pH}$ values of minced meat samples were affected by ginger powder addition. The $\mathrm{pH}$ values decreased when the amount of ginger powder incorporation on minced meat samples increased from 1 to $5 \%$. Moreover, when storage times increased from 0 to 6 days, the treated samples with ginger powder did not showed more changes in terms of $\mathrm{pH}$ values compared to control samples. The overall results of the current study showed that ginger powder addition on meat samples has an important impact on the shelf life and keeping quality of minced meat samples.

As shown in table (7), TBA is a good indicator for the assessment of quality of meat and degree of lipid oxidation. (Ndaw et al., 2008). This result agree with those of Olatidoye O. P.et al., (2015 ) who 
investigated that ginger extract is considered as an important target to investigate in order to provide a new source of natural antioxidants and/or antimicrobial agents. The addition of ginger extracts was significantly effective in reducing thiobarbituric acid reactive substances (TBARS) levels, volatile basic nitrogen (VBN) and total acidity \% relative to control sample during the storage period. The TBA value accepted till $0.9 \mathrm{mg}$ Melanoaldehyde /Kg raw minced beef. All samples were accepted according to (EOS, $\underline{2005}$ ).

Moreover, TVN measurement is the traditional chemical mean most widely used for evaluation of degree of meat spoilage. The obtained results agree with Roundsa et al. ( 2013). they reported that due to antimicrobial effect of olive leaf, ginger and turmeric , reduction and inhibition of the bacteria growth on meat, so it reduces the TVN due to destruction of protein during meat spoilage. The TVN value accepted till $20 \mathrm{mg} / 100 \mathrm{~g}$ in raw minced beef. All the examined samples of both treated and untreated groups were accepted according to (EOS, 2005).

\section{CONCLUSION}

In conclusion, olive leaves extract , curcuma extract and ginger extract have both of antimicrobial and antioxidant effects. olive leaves extract proved to be more efficient . So, the use of natural extracts as it is safe natural agent, is therefore recommended to improve safety and extend shelf life of meat product.

\section{REFERENCES}

Amal, S.H., Soher, E.A.,( 2010): Role of lactic acid bacteria as a biopreservative agent of Talbina. J of American Sci 6: 889-898.

Aggarwal, B.B, Sundaram ,C., Malani, N.and Ichikawa, H. (2007): Curcumin: the
Indian solid gold. Advances in Experimental Medicine and Biology, 595: $1-75$.

Aytul, K.K.( 2010) : Antimicrobial and antioxidant activities of olive leaf extract and its food applications. Turkey, Ms. Thesis, Graduate School of Engineering and Sciences of Izmir Institute of Technology.

Abdollahzadeh E., Rezaei M., Hosseini H. (2014): Antibacterial activity of plant essential oils and extracts: The role of thyme essential oil, nisin, and their combination to control Listeria monocytogenes inoculated in minced fish meat. Food Control, 35: 177183.

Benavente-Garcia, O.; J. Castillo, J. Lorente, A. Ortuno, J.A. Del Rio. (2000): Antioxidant Activity of Phenolics Extracted from Olea europaea $L$. Leaves. Food Chem. 68: 457-462.

Biesalski, H.K., 2005. Meat as a component of a healthy diet - are there any risks or benefits if meat is avoided in the diet? Meat science 70: 509-524.

Briante, R.; La Clara, F.; Tonziello, M.P.; Febbraio, F.; Nucci, R. (2002): Antioxidant Activity of the Main Bioactive Derivatives from Oleuropein Hydrolysis vy Hyperthermophilic $\beta$-Glycosidase. $J$. Agric. Food Chem., 49: 3198-3203.

Calo J. R., Crandall P. G., O'Bryan C. A., Ricke S. C. (2015). Essential oils as antimicrobials in food systems-A review. Food Control. , 54 :111-119. 10.1016/j.foodcont.2014.12.040.

Du, H., and Li, H. (2008): Antioxidant effect of cassia essential oil on deep-fried beef during the frying process. Meat Science, 78: 461-468.

EOS, 2005. (Egyptian Organization for Standardization and Quality 2005. Egyptian Standard, ES. 
FAO, (1980): Food and Agriculture Organization. Manual of food quality control. 4. Microbiological Analysis, FAO, United Nations, Rome. Int. J. Food Microbiol., 80: 241-250.

Fijelu Frank, Yanshun Xu, Qixing jiang and Wenshui Xia (2014) : Protective effects of garlic (Allium sativum) and ginger (Zingiber officinale) on physicochemical and microbial attributes of liquid smoked silver carp (Hypophthalmichthys molitrix) wrapped in aluminium foil during chilled storage, African Journal of Food Science ,8(1):1-8.

Gomez, K.A., Gomez, A.A., (1984): Statistical procedures for agriculture research. . John Wiliy and Sons Editor Inc. USA (2Ed.), Chapter 3: 129-184.

Gork, E.P., (1976): Uber die Ursachen von Qualitatstmangeln bei tiefgeforenen Fertiggerichten auf fleischbasis in der Fluggastverpflegung. . Vet. Med. Diss., tech. Univ. Berlin.

Haq, M. ; Dutta, P.L. ; Sultana, N. and Rahman, A. (2013): Production and quality assessment of fish burger from the grass carp, Ctenopharyngodon idella (Cuvier and Valenciennes, 1844), Journal of Fisheries, 1(1): 42-47.

International Commission and Microbiological Specification for Food "ICMSF" (1978): Microorganism in foods. Their significance and methods of enumeration. 1st, 2nd Ed Toronto, Univ. of Toronto press. Toronto Ontario, . Canada. J.Hyg., 33: 165-174.

International commission of Microbiological Specification for Foods"ICMSF" (1996): Microorganisms in Food. ITheir Significance and methods of enumeration. $3^{\text {rd }}$ Ed. Univ. of Toronto, Canada.

Kumar GS, Harish N, Shyaja MD, Salimath PV (2006) Fress and bound phenolic anti-oxidant in amla (Emblica officinalis ) and turmer (Curcuma longa). J Food Compos Anal ,19:446-452

Lambert, A.D., Smith, J.P., Dodds, K.L., 1991. Shelf life extension and microbiological safety of fresh meat. A review. Food Microbiol. 9: 267-297.

Lee O. H., Lee B. Y. (2010): Antioxidant and antimicrobial activities of individual and combined phenolics in Olea europaea leaf extract. Bioresour. Technol. $101 \quad 3751-3754$. 10.1016/j.biortech.2009.12.052

[PubMed] [Cross Ref].

Mancini, S., Paci, G., Pisseri, F. and Preziuso, G. (2017): Effect of dietary turmeric powder (Curcuma longa L.) on cooked pig meat quality. International Food Research Journal 24(6): 2460-2465 .

Marangoni, C.; Cichoski, A.J. and Barin, J.S (2017) : Effect of olive leaves on the quality of chicken meat during frozen storage. International Food Research Journal 24(1): 164-172.

Mielnik MB, Signe S, Bjorg E, Grete S. (2008): By-products from herbs essential oil production as ingredient in marinade for turkey thighs. Food Science Technology, 41:93-100.

Ndaw, A.D., Faid, M., Bouseta, A., Zinedine, A., (2008): Effect of controlled lactic acid bacteria fermentation on microbiological and chemical quality of Morocans sardines (Sardina pilchardus). Int. J. of Agric. and biology ,10: 21-27.

Olaoye, O.A., Onilude, A.A., 2010. Investigation on potential use of biological agents in the extension of fresh beef in Nigeria. . World $\mathrm{J}$ of Microbiol. and biotechnol. 26: 14451424.

Olatidoye, O. P., Sobowale ,S. S., Oluwafemi, R. A., Alabi, A. O.(2015) : Effects of Adding Ginger Extracts (Zingiber officinale) on Minced Cow Meat During Refrigerated Storage. 
American Journal of Food Science and Nutrition Research. , 2, ( 6): 165-171.

Omojola, A. B. and Adediran, O. A. (2015) :Effect of ethanolic extract of garlic, roselle and ginger on quality of chicken patties. International Food Research Journal 24(6): 2460-2465 .

Pattaratanawadee, E., Thongson, C., Mahakarnchanakul, W., Wanchaitanawong, P. (2006). Antimicrobial activity of spice extracts against pathogenic and spoilage microorganisms. In The proceedings of the 44th Kasetsart University annual conference, Kasetsart, 30 January-2 February, (pp. 297-307). Subject: Agroindustry, Economics, Business Administration.

Pearson, D., Tauber, (1984): Chemical Analysis of Food. 8th Ed. Publishing Co. Churchill Livingstone,s, Edinburgh, London, UK.

Roundsa L, Havensa CM, Feinsteinb Y, Friedmanc $\mathrm{M}$ and Ravishankar $\mathrm{S}$ (2013). Concentration-dependent inhibition of Escherichia coli O157:H7 and heterocyclic amines in heated ground beef patties by apple and olive extracts, onion powder and clove bud oil. Meat Science, 94(4): 461-467.

Shah, M. A. ; Bosco, S. J. D. and Mir, S. A. :(2014) Plant extracts as natural antioxidants in meat and meat products. Meat Science, 98: 21-33.

Sharma, R.A., Gescher, A.J., Steward, W.P. (2005): Curcumin: The story: so far. Euopean Journal of Cancer, 41:19551968.
Skerget, M. Kotnik P., Hadolin, M., Hras A.R., Knez Z., (2005):Phenols, proanthocyanidins, flavons and flavonols in some plant materials and their antioxidant activities. Food Chemistry, 89: 191-198.

Soni, M.G.; Burbock, G.A.; Christian, M.S.; Bitler, C.M.; Crea, R. (2006): Safety Assessment of Aqueous Olive Pulp Extract as an Antioxidant and Antimicrobial Agent in Foods. Food and Chem.Toxic., 44: 903-915.

Tilak JC, Meenal B, Hari M, Devasagayam TPA. (2004): Antioxidant availability of turmeric in relation to its medicinal and culinary uses. Phytother Res.;18:798-804. doi: 10.1002/ptr.1553. [PubMed] [Cross Ref].

Uhart, M., Maks, N., Ravishankar, S. (2006): Effect of spices on growth and survival of salmonella typhimurium dt 104 in ground beef stored at 4 and 8c. J. Food Saf., 26(2):115-125.

Vyncke , W . (1970 ) : Direct determination of thiobarbituric acid value in trichloroacetic acid extract of fish as a measure of oxidative rancidity. Fette Seifen Anstri-climitted. ,72 (12 ) : 1084 - 1087 .

Zemenu Kerie Terefe (2017) : Effect of Ginger (Zingiber officinale) Powder Addition on $\mathrm{pH}$, Titratable Acidity and Total Viable Bacterial Counts of Minced Meat under Refrigerated Storage . International Journal of Food Science and Nutrition Engineering, 7(2): $\quad 38-42 \quad$ DOI: 10.5923/j.food.20170702.03. 